\title{
Overexpression of BTG2 suppresses growth, migration, and invasion of human renal carcinoma cells in vitro
}

\author{
JIN SIMA ${ }^{1,2}$, B. ZHANG ${ }^{1,2, *}$, X. Y. SIMA ${ }^{2}$, Y. X. MAO ${ }^{2}$ \\ ${ }^{1}$ Department of Urology, Aerospace Center Hospital, No.15 Yu-quan Road, Beijing, 100049, China; ${ }^{2}$ Key Laboratory of Oncogenesis and Related \\ Genes Research, Aerospace Clinical Medical College of Peking University, No.15 Yu-quan Road, Beijing, 100049, China
}

*Correspondence: baoztj@sina.cn

Received August 22, 2015/ Accepted December 15, 2015

\begin{abstract}
The objective of the study was to investigate the impact of BTG2 on growth, migration and invasion of human clear cell renal cell carcinoma (ccRCC) cells. Endogenous expression of BTG2 was evaluated in the ccRCC cell lines (Caki-1, 786-O and Caki-2) and noncancerous human renal proximal tubular cell lines (HKC, HK-2 and RPTEC). BTG2 expression was decreased in the ccRCC cells compared with the noncancerous cells $(P<0.01)$. Then Caki-1 and 786-O cells described as suitable transfection hosts were used in transfection to carry out biological function studies. The three experimental groups were as follows: BTG2-ORF (transfected with BTG2-ORF plasmid), blank-Vector (transfected with pCMV6-Entry), and Cell-alone group (no DNA transfected in). BTG2 expression in the BTG2-ORF groups was significantly higher than that in the controls $(P<0.01)$. Cell growth was remarkably reduced and the number of migrating or invading cells was reduced in the BTG2-ORF groups compared with the controls $(P<0.01)$. Furthermore, Matrix Metalloproteinase-9 (MMP-9), Cyclin D1 and Cyclin E expression were reduced in the BTG2-ORF groups compared with the controls. Here, we have provided data for attenuated BTG2 expression in the ccRCC cells. Overexpressed BTG2 could inhibit cell proliferation, migration and invasion of human ccRCC, and the suppressive effects might be due to down-regulation of MMP-9, Cyclin D1 and Cyclin E expression.
\end{abstract}

Key words: BTG2, cell proliferation, cell migration inhibition, neoplasm invasiveness, clear cell renal carcinoma

Renal cell carcinoma (RCC) is the second leading cause of death in patients with urological tumors [1]. Clear cell renal cell carcinoma (ccRCC), which accounts for approximately $75 \%$ of RCC, represents the most common histological subtype [2]. Compared with other malignant neoplasm, few biomarkers for ccRCC have been identified for clinical diagnosis, therapy or outcome prediction $[3,4]$. Several molecular targets were recently suggested as potential solutions, however, the curative effects of these targets remain relatively limited [4-6]. To develop effective diagnostic, preventive, and therapeutic methods, further studies on the pathogenesis of ccRCC are required. Tumorigenesis is the result of the accumulation of multiple mutations, including activation of oncogenes and inactivation of tumor suppressors [7]. Tumor-related genetic changes usually result in several events critical to tumor occurrence and development, such as aberrant alternation of cell proliferation [8]. Numerous studies suggested that inactive onco-suppressors might play a key role in tumor occurrence and development [9-13].
BTG2 is the abbreviation of B cell translocation gene 2, which is the first member identified in the BTG/TOB gene family [14]. There are two conserved and highly homologous domains in the N-terminal of each BTG/TOB family member, named respectively Box A and Box B. The Box A has a main effect on anti-proliferation, while the box B plays a role in interacting with other target molecules $[14,15]$. Because of the anti-proliferative Box A, BTG2 gene is also known as the anti-proliferative gene [15]. Increasing evidences have demonstrated that aberrant BTG2 gene is involved in a variety of tumor types [16-20], such as gastric cancer [16], bladder cancer [17], breast cancer [18], Non-small cell lung cancer (NSCLC) [19] and prostate cancer [20].

In our previous research, a profile was performed using human primary ccRCC tissues and adjacent non-tumor tissues obtained from Chinese patients. The result showed that aberrant BTG2 expression might be a related molecular event in human ccRCC (unpublished data). Up to now, little is known regarding the impact of BTG2 gene on cell growth, 
cell migration and invasion of human ccRCC cells. In the present study, a logical linking was established between BTG2 overexpression and biological characteristics of human ccRCC cells. The abilities of cell growth, cell migration and invasion were assessed, and the effects of BTG2 on expression of Matrix Metalloproteinase-9 (MMP-9), Cyclin D1 and Cyclin E were explored.

\section{Materials and methods}

Cell lines and cell culture. The human ccRCC cell lines (Caki-1, 786-O and Caki-2) and noncancerous human renal proximal tubular cell lines (HKC, HK-2 and RPTEC) were obtained from the Chinese National Platform of Experimental Cell Resources for Sci-Tech (Beijing, China). The ccRCC cells and HKC cells were maintained in RPMI-1640 medium (Gibco, Gaithersburg, MD, US) with $10 \%$ fetal bovine serum (Gibco) and $1 \%$ antibiotics (Invitrogen, Carlsbad, CA, US) in a sterile incubator at $37^{\circ} \mathrm{C}$ with $5 \% \mathrm{CO}_{2}$. The HK-2 and RPTEC cells were cultured at $37^{\circ} \mathrm{C}$ and $5 \% \mathrm{CO}_{2}$ in renal epithelial cell basal medium (REBM) supplemented with REBM complex (Lonza, Inc., Walkersville, MD, US). Cells at logarithmic phase were collected for the experiments.

Plasmid transfection. The human cDNA-ORF clone of BTG2 gene (BTG2-ORF plasmid), blank vector (pCMV6Entry) and transfection reagent MegaTran 1.0 were purchased from OriGene Technologies, Inc. (Rockville, MD, US). The experimental cells were divided equally into three groups as follows: BTG2-ORF (transfected with BTG2-ORF plasmid), blank-Vector (transfected with pCMV6-Entry), and Cell-alone group (no DNA transfected in). The day prior to transfection, cells were seeded at a density of $2 \times 10^{5} /$ well into 6 -well plates. Cells were transfected with BTG2-ORF plasmid or vector for $6 \mathrm{~h}$ in serum-free medium Opti-MEM I (Gibco) according to the manufacturer's instructions. After $24 \mathrm{~h}$, the transfected cells were diluted at 1:15 into 12-well plates and the culture medium was replaced with complete medium containing G418 $(800 \mu \mathrm{g} / \mathrm{ml})$. The stable positive clones were obtained

Table 1. Specific primers for PCR analysis

\begin{tabular}{llc}
\hline Gene & Primer sequences & $\begin{array}{c}\text { Amplicon } \\
\text { (bp) }\end{array}$ \\
\hline BTG2 & F: 5'-CATCATCAGCAGGGTGGC-3' & 115 \\
& R: 5'-CCCAATGCGGTAGGACAC-3' & \\
MMP-9 & F: 5'-TTGACAGCGACAAGAAGTGG-3' & 148 \\
& R: 5'-CCCTCAGTGAAGCGGTACAT-3' & \\
Cyclin D1 & F: 5'-CCCTCGGTGTCCTACTTCAA-3' & 149 \\
& R: 5'-AGGAAGCGGTCCAGGTAGTT-3' & \\
Cyclin E & F: 5'-TTCTTGAGCAACACCCTCTTCTGCA- & 138 \\
& GCC-3' & \\
& R: 5'-TCGCCATATACCGGTCAAAGAAATCTT- \\
PPIA & GTGCC-3' & \\
& F: 5'-TTCATCTGCACTGCCAAGAC-3' & 158 \\
& R: 5'-TCGAGTTGTCCACAGTCAGC-3' & \\
\hline
\end{tabular}

after 21 days of screening by G418, and protein expression was evaluated using western blot.

Real-time quantitative PCR analysis. Total RNA was extracted using Trizol reagent (Invitrogen) and reverse transcribed to cDNA using the SuperScriptTM II Reverse Transcriptase Kit (Invitrogen) according to the manufacturer's instructions. Real-Time quantitative polymerase chain reaction (PCR) was performed using the standard SYBR Green I PCR Kit (Fermentas, Pittsburgh, PA, US) in an ABI 7500 Sequence Detection System (Applied Biosystems, Foster City, CA, US). The expression of mRNA was determined from the threshold cycle values, and the relative expression levels were normalized to the expression of human peptidylprolyl isomerase $A$ (PPIA) and calculated by the $2^{-\triangle \Delta C T}$ method as described in the literature [21]. Primers used in PCR are listed in Table 1. The experiments were repeated three times and every sample was performed in triplicate.

Western blot. Western blot was performed following standard techniques. Protein lysates were extracted using RIPA lysis buffer (Santa Cruz Bio-tech, Santa Cruz, CA, US) containing proteinase inhibitors. BCA reagent (Applygen Tech., Beijing, China) was used to quantify the protein concentration. Equivalent amounts of protein $(50 \mu \mathrm{g} /$ lane $)$ were fractionated by $10 \%$ sodium dodecyl sulfate polyacrylamide gels electrophoresis and transferred to a polyvinylidene fluoride membrane (Millipore, Billerica, MA, US). The membrane was then blocked in $0.1 \%$ Triton X-100 and 5\% non-fat milk powder in phosphate-buffered saline (PBS) for $2 \mathrm{~h}$, and then probed with primary antibodies at $4^{\circ} \mathrm{C}$ overnight. Rabbit polyclonal primary antibodies against BTG2, MMP-9, Cyclin D1 and Cyclin E (Abcam, Cambridge, MA, US) were used at a dilution of 1:500. Anti-GAPDH, a mouse monoclonal antibody (Santa Cruz Bio-tech) at a 1:1000 dilution, was used as a house keeping gene. The membrane was incubated with secondary antibody at a 1:2500 dilution (Santa Cruz Bio-tech) for $1 \mathrm{~h}$ at room temperature and the bands were visualized by a Chemiluminescence Detection Kit (Pierce, Rockford, IL, US) using medical X-ray films (Kodak, Rochester, NY, US) and quantified by Photoshop CS5 (Adobe Software, San Jose, CA, US). The relative expression of the target gene was normalized to GAPDH levels.

MTS assay. MTS reagent (Promega, Madison, WI, US), an aqueous cell proliferation assay, was used to assess cell growth. The experimental cells were seeded into 96-well plates with $2 \times 10^{3}$ cells/ well. At each check-point $(0,24,48,72$ or $96 \mathrm{~h})$, $20 \mu \mathrm{l}$ of MTS reagent was directly added into each well and cells were incubated at $37^{\circ} \mathrm{C}$ for $1 \mathrm{~h}$. The absorbance at 490 $\mathrm{nm}(A 490)$ was measured by a 96 -well microplate reader. The experiment was repeated three times with four replicates in each check-point.

Flow cytometry. Cells were cultured in regular medium until $70 \%-80 \%$ confluence, switched to serum-free medium for $24 \mathrm{~h}$ to synchronize. Cells were collected and washed with PBS, and fixed with $70 \%$ cold ethanol at $4{ }^{\circ} \mathrm{C}$ overnight. The fixed cells were resuspended in ice-cold PBS and stained with 
propidium iodide (BD Biosciences, San Jose, CA, US) according to the manufacturer's instructions. The stained cells were analyzed on the FACS-Caliber using CellQuestPro software, and the proportion of cells at $G_{0} / G_{1}$ phase, $S$ phase or $G_{2} / M$ phase was calculated. Each assay was performed independently three times.

Transwell assay. Cell migration or invasion assay was carried out using Boyden chambers containing a Transwell membrane filter with a pore size of $8 \mu \mathrm{m}$ (Corning Costa Corp., Cambridge, MA, US). Prior to the invasion assay, the filter membrane was coated with $60 \mu \mathrm{l}$ of Matrigel (BD Biosciences) at a 1:8 dilution and rehydrated by adding $0.5 \mathrm{ml}$ serum-free medium to the upper chambers at $37^{\circ} \mathrm{C}$ for $0.5 \mathrm{~h}$. The experimental cells $\left(3.5 \times 10^{4}\right.$ cells/ well for invasion assay or $8 \times 10^{4}$ cells/ well for migration assay) were added to the upper chamber with $200 \mu$ culture medium, and the lower chamber was filled with $500 \mu \mathrm{l}$ medium containing $10 \%$ fetal bovine serum. After $6 \mathrm{~h}$ (for migration assay) or $12 \mathrm{~h}$ (for invasion assay) at $37^{\circ} \mathrm{C}$ incubation, the surface of the filter was swept by cotton swabs. The membrane was fixed with methanol and stained with crystal violet, and cells were counted under a microscope in five random fields, irrespective of staining intensity. Each experiment was repeated three times.

Statistical analysis. The data were expressed as mean \pm standard deviation and analyzed using the SPSS statistical software 13.0 (SPSS Inc., Chicago, IL, US). A Student's t test was used for the comparison of two groups, and one-way ANOVA for multiple groups. A value of $P<0.05$ was considered as statistically significant.

\section{Results}

Attenuation of BTG2 expression was observed in the ccRCC cells. To determine the role of BTG2 in ccRCC, we first detected endogenous BTG2 expression in human normal kidney and ccRCC cell lines. The relative mRNA levels of BTG2 in the ccRCC cells were significantly down-regulated compared with the noncancerous cells $(P<0.01$, Fig. 1a). As expected, reduced BTG2 protein bands were observed in the ccRCC cells $(P<0.01$, Fig. 1b). These indicated that attenuation of BTG2 expression might be a causative factor correlated with the carcinogenesis of ccRCC.

Abundant BTG2 was detected in the BTG2-ORF groups. BTG2 expression was examined in the three experimental groups. We observed apparently high levels of BTG2 in the BTG2-ORF groups at both protein and mRNA levels compared with the controls $(P<0.01$, Fig. 2$)$. These data confirmed up-regulation of BTG2 in the BTG2-ORF groups of Caki-1 and 786-O cells.

Overexpression of BTG2 suppressed cell growth. To determine whether overexpression of BTG 2 affected cell growth, MTS assay was performed to assess cell viability and proliferation. Cell viability was analyzed by comparing OD values at $490 \mathrm{~nm}$ of the three groups at $48 \mathrm{~h}$ or $72 \mathrm{~h}$, and cell growth curves were plotted using the average of $A 490$ values at each time point. The data demonstrated that cell viabilities in the BTG2-ORF group of Caki-1 and that of 786-O cells were both lower than those of their control groups $(P<0.01$, Fig. 3a-3b), and there was no significant difference between the two controls $(P>0.05)$. As shown in Fig. 3c-3d, the cell growth curves showed that cell proliferation in the BTG2-ORF group of Caki-1 and that of 786-O cells were both reduced compared with their controls.

Overexpression of BTG2 induced $G_{0} / G_{1}$ cell cycle arrest. To determine the effects of BTG2 on cell cycle, flow cytometry was performed. The percent of cells at $\mathrm{G}_{0} / \mathrm{G}_{1}$ phase in the BTG2-ORF group was higher than the two controls $(P<0.01$, Fig. $4 \mathrm{a}-4 \mathrm{~b})$. Furthermore, the $\mathrm{S}$ phase fraction was decreased in the BTG2-ORF group compared with the controls $(P<0.01$, Fig. $4 \mathrm{c})$. These data suggested that overexpression of BTG2 induced cell cycle arrest at the $G_{1} / S$ phase transition. The proliferation index was calculated as $\left(\mathrm{G}_{2} / \mathrm{M}+\mathrm{S}\right) /\left(\mathrm{G}_{0} / \mathrm{G}_{1}+\mathrm{S}+\mathrm{G}_{2} / \mathrm{M}\right)$. The proliferation index of the BTG2-ORF group was remarkably decreased compared with the controls $(P<0.01$, Fig. $4 \mathrm{~d})$. These data were additional

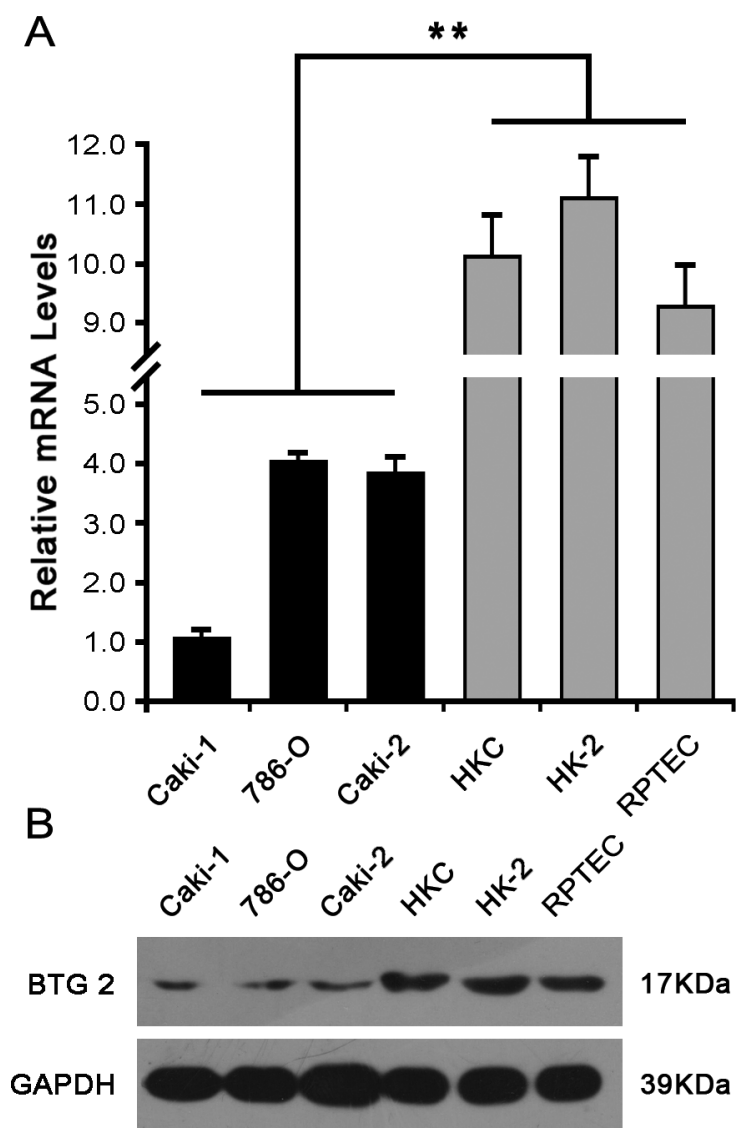

Figure 1. The endogenous expression of BTG2 in the ccRCC cells (Caki-1, 786-O, Caki-2) and non-cancerous cells (HKC, HK-2 and RPTEC). (a) The mRNA levels of BTG2 in the ccRCC cells were down-regulated compared with those of the non-cancerous cells. (b) The reduced BTG2 protein expression was observed in the ccRCC cells. ${ }^{* *} P<0.01$ 
A
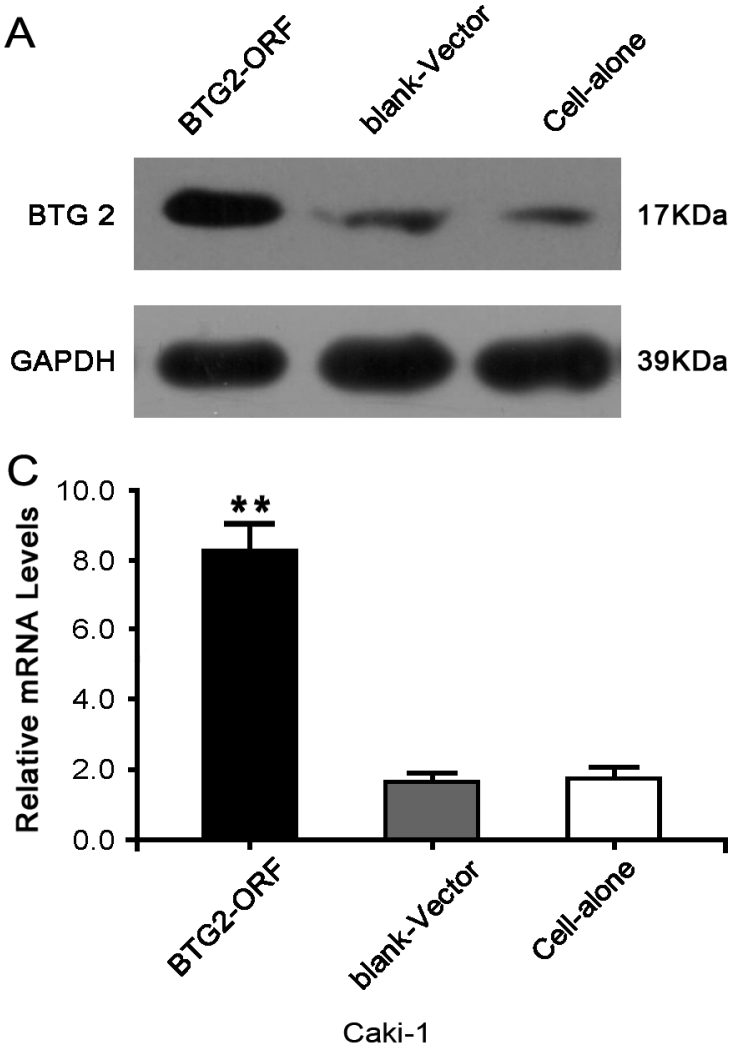

B
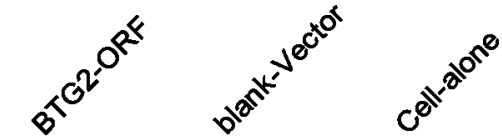

BTG 2

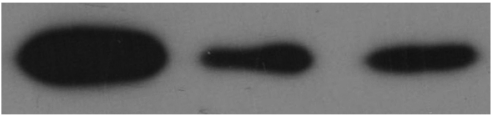

$17 \mathrm{KDa}$
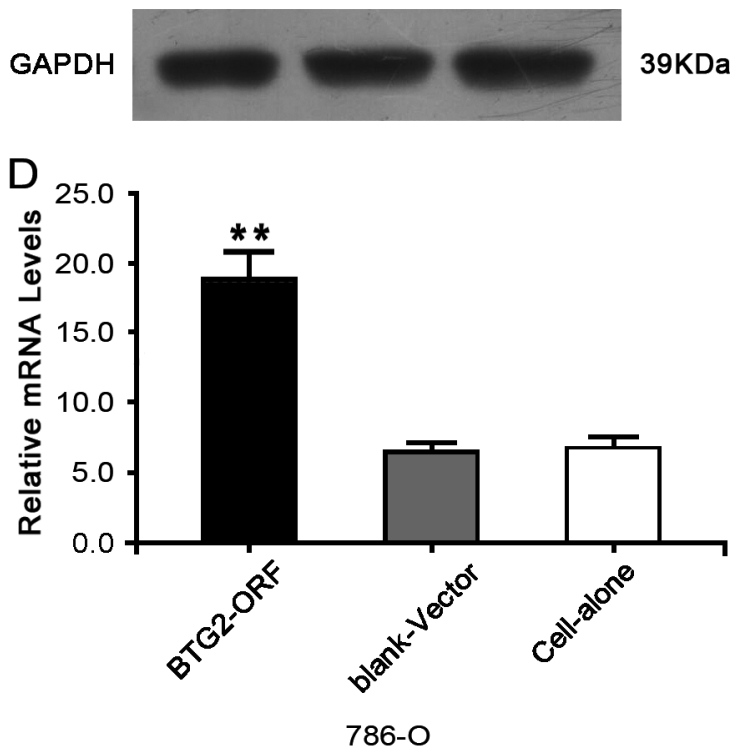

Figure 2. The expression of BTG2 in the three experimental groups. (A) There is an abundant protein expression of BTG2 in the BTG2-ORF group of Caki-1 cells. (B) The mRNA level of BTG2 was up-regulated in the BTG2-ORF group of Caki-1 cells. (C) There is an abundant protein expression of BTG2 in the BTG2-ORF group of 786-O cells. (D) The mRNA level of BTG2 was up-regulated in the BTG2-ORF group of $786-\mathrm{O}$ cells. ${ }^{* * P}<0.01$

proofs to that overexpressed BTG2 suppressed proliferation of human ccRCC cells.

Overexpression of BTG2 inhibited invasive ability and metastatic potential. Transwell assays were performed to study the impact of BTG2 gene on invasive and migratory abilities in Caki-1 and 786-O cells. Both in migration and invasion assays, crystal violet-staining showed that the number of cells passing through the polycarbonate membranes in the BTG2-ORF groups was significantly less than their controls $(P<0.01$, Fig. 5). There were no statistically significant differences between the two controls $(P>0.05)$.

Effects of BTG2 on expression of MMP-9, Cyclin D1 and Cyclin E. The expression of MMP-9, Cyclin D1 and Cyclin $\mathrm{E}$ were detected by western blot. The protein bands analysis demonstrated that expression of MMP-9, Cyclin D1 and Cyclin $\mathrm{E}$ in the BTG2-ORF group of Caki-1 and that of 786-O cells were both down-regulated, compared to that in their controls $(P<0.01$, Fig. 6).

\section{Discussion}

Kidney cancer is a common urological therioma $[1,2]$. Currently, partial or radical nephrectomy is the most common effective treatment for localized ccRCC [22]. Unfortunately, many patients who suffer from ccRCC developed metastases at the time of diagnosis and lost the opportunity of operation $[23,24]$. What is worse, most of these advanced or metastatic ccRCC patients are not sensitive to chemotherapy and radiotherapy, and therefore, their prognosis is extremely poor $[5,24]$. Some targeted-therapy drugs, such as sorafenib and sunitinib, show substantial effects on patients with advanced or metastatic ccRCC. However, complete responses and curative effects of these treatments are rarely observed $[25,26]$. Consequently, appropriate biomarkers and therapeutic targets of ccRCC are still needed.

BTG2 is considered as an early growth response gene [14]. The multifunctional BTG2 protein is involved in cell proliferation, cell maintenance and others [14-16]. Recently, several findings indicate that, aside from regulating such physiologic developmental processes, BTG2-dependent events play a critical role in human cancerogenesis [16-20]. Early studies have shown that aberrant BTG2 has been involved in tumor development and progression. Takahashi et al. [18] demonstrated that decreased expression of BTG2 protein was observed in estrogen receptor-positive breast cancer, and was associated with high tumor grade, size, and 

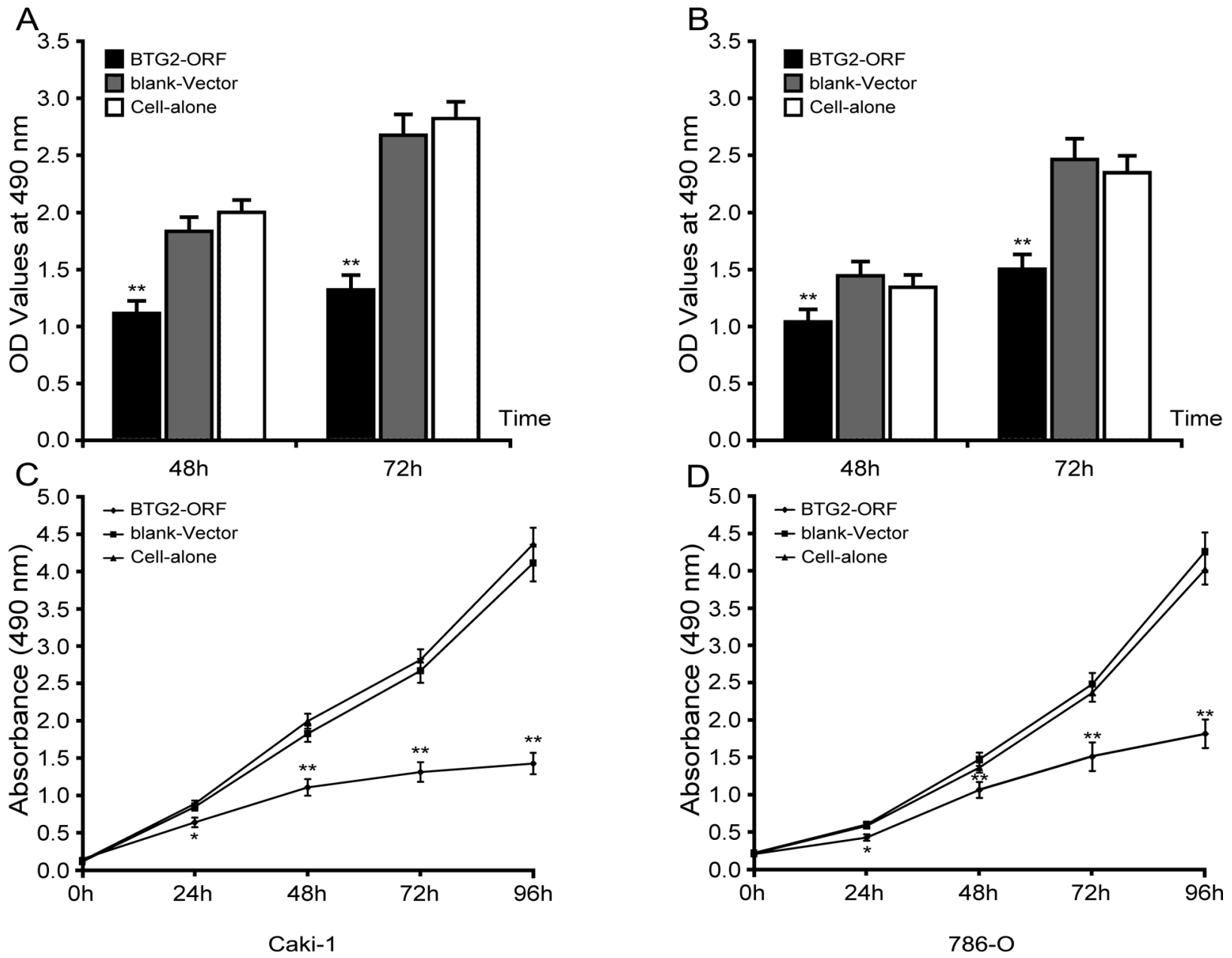

Figure 3. The effects of BTG2 on cell growth in vitro. (A) Cell viability was analyzed by comparing OD values at $490 \mathrm{~nm}$, and $\mathrm{OD}_{490}$ values in the BTG2-ORF group of Caki-1 cells were lower than those in the controls. (B) OD $_{490}$ values in the BTG2-ORF group of 786-O cells were lower than those in the controls. (C) Cell growth curves were plotted using the average of $A_{490}$ at each check point, and cell proliferation in the BTG2-ORF group of Caki-1 cells was weakened. (D) Cell proliferation in the BTG2-ORF group of 786-O cells was weakened compared with the controls. ${ }^{\star} P$ $<0.05$ and ${ }^{* *} P<0.01$

recurrence. In vitro assays, knockdown of BTG2 expression promotes cell growth, cell motility, and distal metastasis of breast tumor [27]. Sun et al. [28] reported a frequent loss of BTG2 expression in several human lung carcinomas cell lines. And overexpression of BTG2 can inhibit cell growth, invasive ability and metastatic potential of NSCLC cells [19]. However, Wagener et al [17] showed that BTG2 gene promoted the migration of bladder cancer cells and overexpressed BTG2 is associated with poor survival in patients with bladder cancer. From above, the roles of BTG2 in oncogenesis may be cancer type-dependent, BTG2 functions as a tumor-suppressor in some human cancers, while it also serves as a tumor-promoter in other human carcinoma [14]. In 2004, a research team from Switzerland analyzed primary ccRCC and non-tumor tissues by using BD Atlas Human Cancer $1.2 \mathrm{cDNA}$ microarrays. Twenty-five genes were found differentially expressed, and BTG2 gene was one of them [29]. However, the effect of BTG2 gene on cellular biological function of ccRCC has not been reported.

In our previous research, a profile showed that BTG2 expression in ccRCC tissues of Chinese patients was reduced more than 70\% compared with non-tumor control references (unpublished data). Based on preliminary findings, a series of experiments were conducted to verify the biological function of BTG2 in ccRCC cells. First, 6 cell lines, including the ccRCC cells (Caki-1, 786-O and Caki-2) and noncancerous human renal proximal tubular cells (HKC, HK-2 and RPTEC), were used to evaluate endogenous expression of BTG2. Significantly decreased BTG2 in the ccRCC cells was observed compared with the references. Next, Caki- 1 and 786-O cells described as suitable transfection hosts in the ATCC website (www.atcc.org, USA) were transfected respectively with 


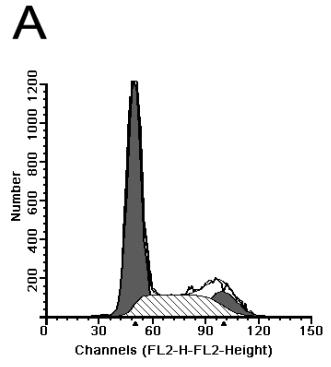

BTG2-ORF

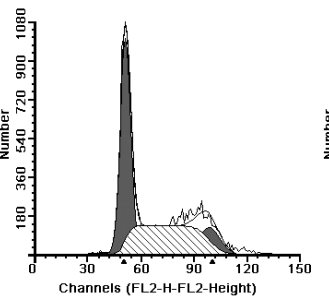

blank-Vector

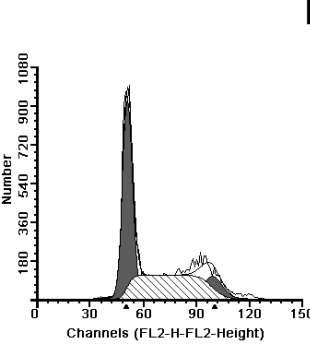

Cell-alone
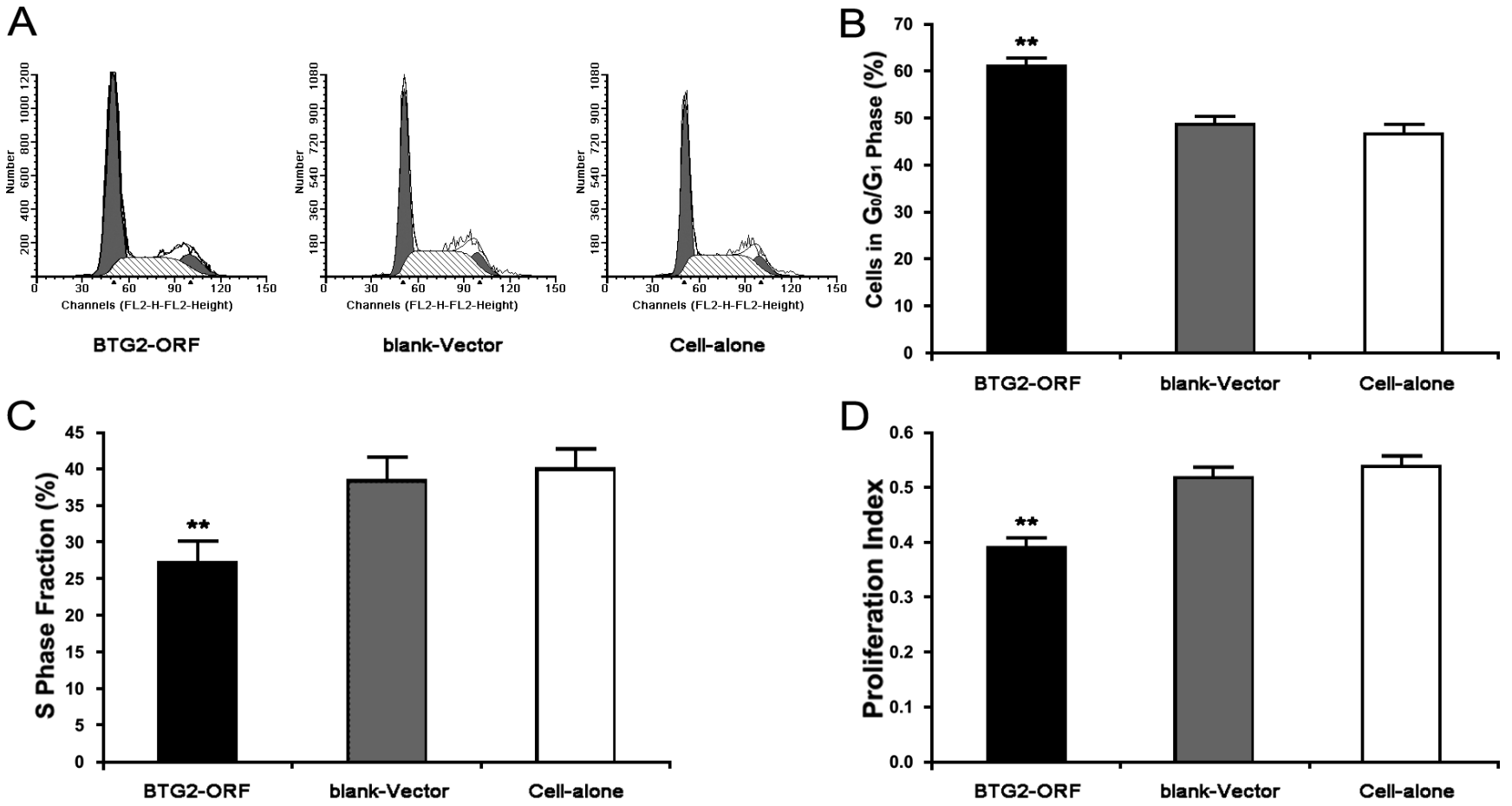

Figure 4. The effects of BTG2 on cell cycle in vitro. (A) Cell cycle distribution was determined with FACS-Caliber, and the representative FACS analyses were shown. (B) The percent of cells at $G_{0} / G_{1}$ phase in the BTG2-ORF group was higher than those in the controls. (C) The $S$ phase fraction of the BTG2-ORF group was reduced compared with the controls. (D) The proliferation index of the BTG2-ORF group was decreased compared with controls. ${ }^{*} P<0.01$

BTG2-ORF plasmid and its vector. We evaluated the effects of overexpressed BTG2 on biological characteristics, including abilities of cell growth, cell migration and invasion. Our data showed that BTG2 overexpression suppressed cell migration and invasion, and also inhibit cell growth. Meanwhile, flow cytometry analysis showed cell cycle arrest at the $G_{1} / S$ phase transition in the BTG2-ORF group, and proliferation index and $\mathrm{S}$ phase fraction was remarkably decreased compared with the controls. These data were additional proofs to cell growth inhibition. Furthermore, MMP-9, Cyclin D1 and Cyclin E expression were reduced accompanied with the BTG2 overexpression.

Up to now, many studies have demonstrated that Cyclin D1 and $\mathrm{E}$ are prevalent regulators involved in cancer development. The association between aggressiveness of tumor growth and expression of Cyclin D1 and Cyclin E has been observed in various tumors [30]. Hedberg et al. [31] examined the protein contents in tissues of human ccRCC, and overexpression of Cyclin D1 and Cyclin E were observed. Similarly, Lima et al. [32] and Nauman et al. [33] showed the high levels of Cyclin D1 and Cyclin E protein in human ccRCC. In cell experiments, researchers found that decreased Cyclin D1 and Cyclin E could inhibit the proliferation of RCC cells $[34,35]$. It is well known that Cyclin D1 and Cyclin E are key regulators in $G_{1}$ phase of cell cycle and they are both rate limiting for the $G_{1}$ to $S$ phase transition [36]. Ohtsubo et al. [37] reported that
Cyclin E is acting after Cyclin D1 in human cell cycle regulation. When Cyclin D1 or/and Cyclin E is overexpressed in cells, the length of $G_{1}$ phase is reduced, which results in an overall faster growth rate. Conversely, down-regulation of Cyclin D1 or/and Cyclin E inhibits the ability of the cells to go into $S$ phase and lead to growth inhibition [38]. MMP-9, which can degrade type III, IV and V collagens, is a member of the MMPs family [39]. Currently known, proteolytic degradation of the basement membrane and extracellular matrix by MMPs is an initial step for tumor invasion and metastasis $[39,40]$. In particular, MMP-9 is consistently expressed in malignant tissues and associated with increased invasiveness in various cancer cells [41]. There has been reported that high MMP-9 expression was associated with prognosis of human ccRCC, and reduced MMP-9 could inhibit invasive potential of human ccRCC cells [40].

In conclusion, here we have provided the evidence of reduced BTG2 expression both at protein and mRNA levels in the human ccRCC cells. Overexpressed BTG2 could inhibit cell proliferation, cell migration and invasion in ccRCC, and the suppressive effects might be due to down-regulation of MMP-9, Cyclin D1 and Cyclin E expression. Taken together, our data implied that BTG2 possibly function as a tumor suppressor in the carcinogenesis of ccRCC, and overexpression of BTG2 expression may an effective treatment for ccRCC patients. 
A
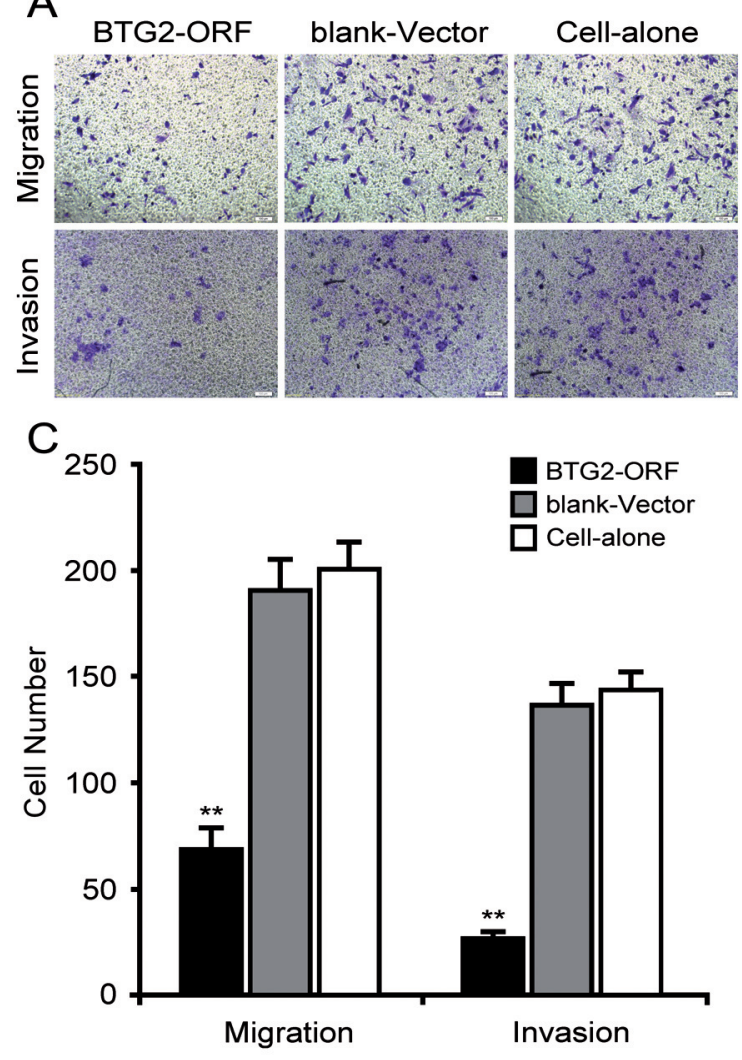

Caki-1
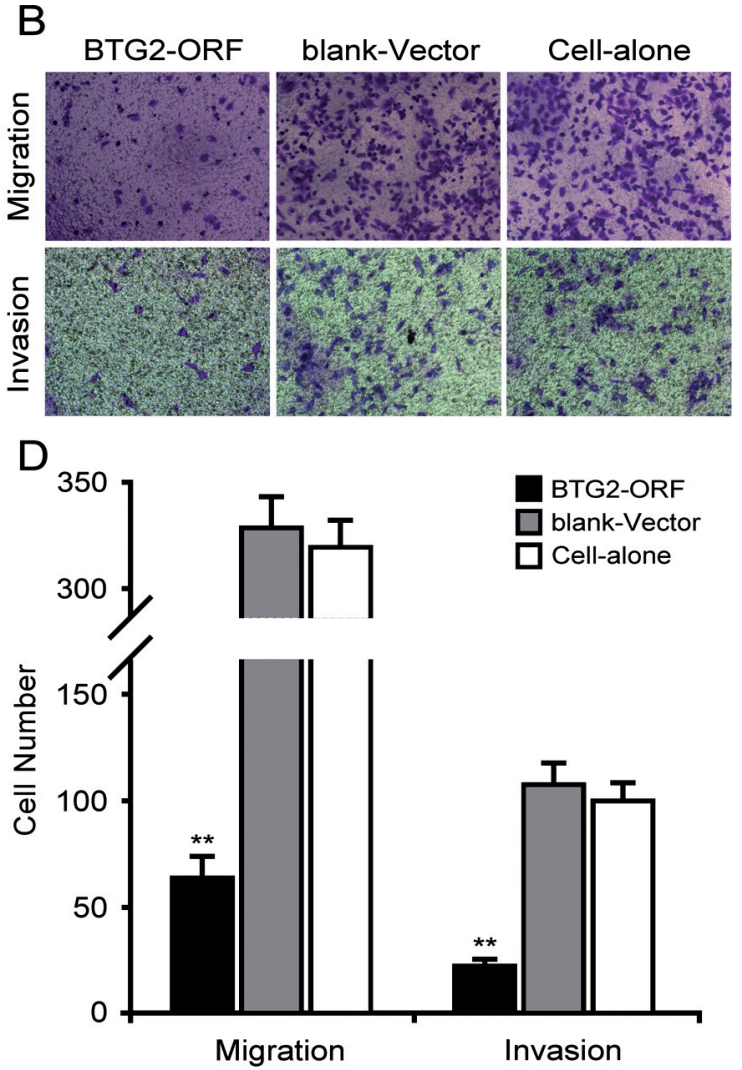

$786-\mathrm{O}$

Figure 5. The impact of BTG2 on migratory and invasive abilities. (A) The representative views of crystal violet-stained Caki-1 cells in migration and invasion Transwell assays. (B) The representative views of crystal violet-stained 786-O cells in migration and invasion Transwell assays. (C) The number of cells in the BTG2-ORF group of Caki-1 cells was less than that in the controls. (D) The number of cells in the BTG2-ORF group of 786-O cells was less than that in the controls. ${ }^{* *} P<0.01$.
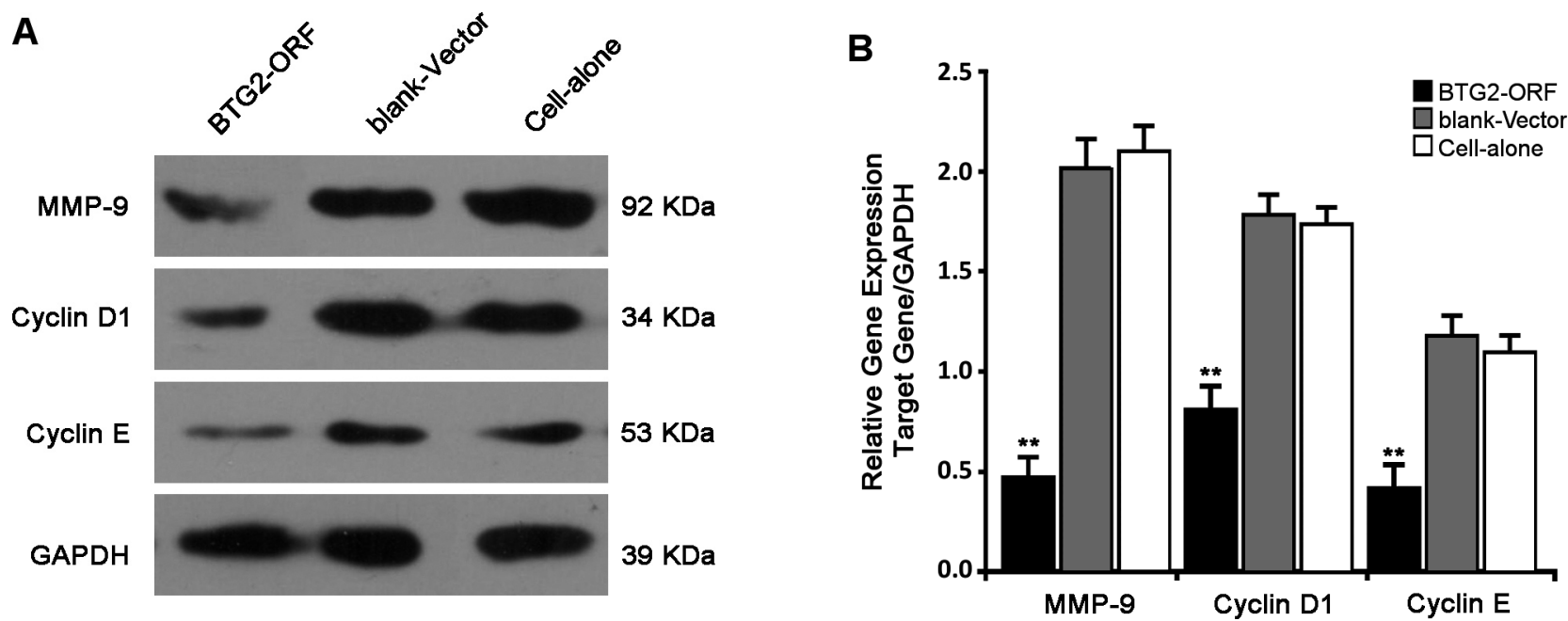

Figure 6. The effects of overexpressed BTG2 on MMP-9, Cyclin D1 and Cyclin E expression. (A) There was an attenuation of MMP-9, Cyclin D1 or Cyclin E expression in the BTG2-ORF group of Caki-1 cells. (B) The relative gene expression of MMP-9, Cyclin D1 and Cyclin E in the three groups of 786-O cells. ${ }^{* *} P<0.01$. 


\section{References}

[1] LYX X, LI H, MA X, LI X, GAO Y, et al. High-Level S100A6 Promotes Metastasis and Predicts the Outcome of T1-T2 Stage in Clear Cell Renal Cell Carcinoma. Cell Biochem Biophys 2015; 71: 279-290. http://dx.doi.org/10.1007/s12013-014$\underline{0196-\mathrm{X}}$

[2] AI Q, MA X, HUANG Q, LIU S, SHI T, et al. High-level expression of Notch1 increased the risk of metastasis in $\mathrm{T} 1$ stage clear cell renal cell carcinoma. PLoS One 2012; 7: e35022. http://dx.doi.org/10.1371/journal.pone.0035022

[3] CASTELLANO D, VIRIZUELA J, CRUZ J, SEPULVEDA J, SAEZ M, et al. The role of pharmacogenomics in metastatic renal cell carcinoma. Cancer Metastasis Rev 2012; 31: S29-32. http://dx.doi.org/10.1007/s10555-012-9356-X

[4] MA X, GAO Y, FAN Y, NI D, ZHANG Y, et al. Overexpression of E2F1 promotes tumor malignancy and correlates with TNM stages in clear cell renal cell carcinoma. PLoS One 2013; 8: e73436. http://dx.doi.org/10.1371/journal.pone.0073436

[5] SONG E, MA X, LI H, ZHANG P, NI D et al. Attenuation of krüppel-like factor 4 facilitates carcinogenesis by inducing G1/S phase arrest in clear cell renal cell carcinoma. PLoS One 2013; 8: e67758. http://dx.doi.org/10.1371/journal. pone. 0067758

[6] ZODRO E, JAROSZEWSKI M, IDA A, WRZESINSKI T, KWIAS Z, et al. FUT11 as a potential biomarker of clear cell renal cell carcinoma progression based on meta-analysis of gene expression data. Tumor Biol 2014; 35: 2607-2617. http:// dx.doi.org/10.1007/s13277-013-1344-4

[7] SIMA J, ZHANG B, YU Y, SIMA X, MAO Y. Overexpression of Numb suppresses growth, migration, and invasion of human clear cell renal cell carcinoma cells. Tumor Biol 2015; 36: 885-892. http://dx.doi.org/10.1007/s13277-014-2918-5

[8] SHI TP, XU H, WEI JF, AI X, MA X, et al. Association of low expression of notch- 1 and jagged-1 in human papillary bladder cancer and shorter survival. J Urol 2008; 180: 361-366. http://dx.doi.org/10.1016/j.juro.2008.02.037

[9] HU F, MENG X, TONG Q, LIANG L, XIANG R, et al. BMP-6 inhibits cell proliferation by targeting microRNA-192 in breast cancer. Biochim Biophys Acta 2013; 1832: 2379-2390. http:// dx.doi.org/10.1016/j.bbadis.2013.08.011

[10] SERTKAYA S, HAMID SM, DILSIZ N, VARISLI L. Decreased expression of EFS is correlated with the advanced prostate cancer. Tumor Biol 2015; 36: 799-805. http://dx.doi.org/10.1007/ s13277-014-2703-5

[11] YOO TH, RYU BK, LEE MG, CHI SG. CD81 is a candidate tumor suppressor gene in human gastric cancer. Cell Oncol 2013; 36: 141-153. http://dx.doi.org/10.1007/s13402-012-0119-Z

[12] LI Q, SHI R, WANG Y, NIU X. TAGLN suppresses proliferation and invasion, and induces apoptosis of colorectal carcinoma cells. Tumor Biol 2013; 34: 505-513. http://dx.doi. org/10.1007/s13277-012-0575-0

[13] SOBTI RC, SINGH N, HUSSAIN S, SURI V, NIJHAWAN R, et al. Aberrant promoter methylation and loss of suppressor of cytokine signalling-1 gene expression in the development of uterine cervical carcinogenesis. Cell Oncol 2011; 34: 533-543. http://dx.doi.org/10.1007/s13402-011-0056-2
[14] MAO B, ZHANG Z, WANG G. BTG2: a rising star of tumor suppressors. Int J Oncol 2015; 46: 459-464.

[15] HU X, XING L, JIAO Y, XU J, WANG X, et al. BTG2 overexpression increases the radiosensitivity of breast cancer cells in vitro and in vivo. Oncol Res 2013; 20: 457-465. http://dx.doi. org/10.3727/096504013X13685487925211

[16] ZHANG L, HUANG H, WU K, WANG M, WU B. Impact of BTG2 expression on proliferation and invasion of gastric cancer cells in vitro. Mol Biol Rep 2010; 37: 2579-2586. http:// dx.doi.org/10.1007/s11033-009-9777-y

[17] WAGENER N, BULKESCHER J, MACHER S, KARAPANAGIOTOU I, HATIBOGLU G, et al. Endogenous BTG2 expression stimulates migration of bladder cancer cells and correlates with poor clinical prognosis for bladder cancer patients. Br J Cancer 2013; 108: 973-982. http://dx.doi. org/10.1038/bjc.2012.573

[18] TAKAHASHI F, CHIBA N, TAJIMA K, HAYASHIDA T, SHIMADA $\mathrm{T}$, et al. Breast tumor progression induced by loss of BTG2 expression is inhibited by targeted therapy with the ErbB/HER inhibitor lapatinib. Oncogene 2011; 30: 3084-3095. http://dx.doi.org/10.1038/onc.2011.24

[19] WEI S, HAO C, LI X, ZHAO H, CHEN J, et al. Effects of BTG2 on proliferation inhibition and anti-invasion in human lung cancer cells. Tumour Biol 2012; 33: 1223-1230. http://dx.doi. org/10.1007/s13277-012-0370-y

[20] JALAVA SE, URBANUCCI A, LATONEN L, WALTERING KK, SAHU B, et al. Androgen-regulated miR-32 targets BTG2 and is overexpressed in castration-resistant prostate cancer. Oncogene 2012; 31: 4460-4471. http://dx.doi.org/10.1038/ onc. 2011.624

[21] SCHMITTGEN TD, LIVAK KJ. Analyzing real-time PCR data by the comparative CT method. Nat Protoc 2008; 3: 1101-1108. http://dx.doi.org/10.1038/nprot.2008.73

[22] MA X, FAN Y, GAO Y, ZHANG Y, HUANG Q, et al. Dicer is down-regulated in clear cell renal cell carcinoma and in vitro Dicer knockdown enhances malignant phenotype transformation. Urol Oncol 2014; 32: e9-17. http://dx.doi. org/10.1016/j.urolonc.2013.06.011

[23] WEI C, WU S, LI X, WANG Y, REN R, et al. High expression of FER tyrosine kinase predicts poor prognosis in clear cell renal cell carcinoma. Oncol Lett 2013; 5: 473-8.

[24] VOGELZANG NJ. Treatment options in metastatic renal carcinoma: an embarrassment of riches. J lin Oncol 2006; 24: 1-3. http://dx.doi.org/10.1200/JCO.2005.03.7234

[25] ESCUDIER B, EISEN T, STADLER WM, SZCZYLIK C, OUDARD $S$, et al. Sorafenib in advanced clear-cell renal-cell carcinoma. N Engl J Med 2007; 356: 125-134. http://dx.doi. org/10.1056/NEJMoa060655

[26] MOTZER RJ, HUTSON TE, CELLA D, REEVES J, HAWKINS $\mathrm{R}$, et al. Pazopanib versus sunitinib in metastatic renal-cell carcinoma. N Engl J Med 2013; 369: 722-731. http://dx.doi. org/10.1056/NEJMoa1303989

[27] ZHANG YJ, WEI L, LIU M, LI J, ZHENG YQ, et al. BTG2 inhibits the proliferation, invasion, and apoptosis of MDAMB-231 triple-negative breast cancer cells. Tumor Biol 2013; 34: 1605-1613. http://dx.doi.org/10.1007/s13277-013$\underline{0691-5}$ 
[28] SUN Q, HANG M, GUO X, SHAO W, ZENG G. Expression and significance of miRNA-21 and BTG2 in lung cancer. Tumor Biol 2013; 34: 4017-4026. http://dx.doi.org/10.1007/ s13277-013-0992-8

[29] STRUCKMANN K, SCHRAML P, SIMON R, ELMENHORST K, MIRLACHER, $M$ et al. Impaired expression of the cell cycle regulator BTG2 is common in clear cell renal cell carcinoma. Cancer Res 2004; 64: 1632-1638. http://dx.doi. org/10.1158/0008-5472.CAN-03-1687

[30] CHRISTOV K, IKUI A, SHIIKAITIS A, GREEN A, YAO R, et al. Cell proliferation, apoptosis, and expression of cyclin $\mathrm{D} 1$ and cyclin $\mathrm{E}$ as potential biomarkers in tamoxifen-treated mammary tumors. Breast Cancer Res Treat 2003; 77: 253-264. http://dx.doi.org/10.1023/A:1021804121171

[31] HEDBERG Y, LJUNGBERG B, ROOS G, LANDBERG G. Expression of cyclin D1, D3, E, and p27 in human renal cell carcinoma analysed by tissue microarray. Br J Cancer 2003; 88: 1417-1423. http://dx.doi.org/10.1038/sj.bjc.6600922

[32] LIMA MS, PEREIRA RA, COSTA RS, TUCCI S, DANTAS $\mathrm{M}$, et al. The prognostic value of Cyclin D1 in renal cell carcinoma. Int Urol Nephrol 2014; 46: 905-913. http://dx.doi. org/10.1007/s11255-013-0602-0

[33] NAUMAN A, TUROWSKA O, POPLAWSKI P, MASTER A, TANSKI Z, et al. Elevated cyclin E level in human clear cell renal cell carcinoma: possible causes and consequences. Acta Biochim Pol 2007; 54: 595-602.

[34] MA JJ, LIAO CG, JIANG X, ZHAO HD, YAO LB, et al. NDRG2 suppresses the proliferation of clear cell renal cell carcinoma cell A-498. J Exp Clin Cancer Res 2010; 29: 103. http://dx.doi.org/10.1186/1756-9966-29-103

[35] WANG P, YIN B, SHAN L, ZHANG H, CUI J, et al. RNA interference-mediated knockdown of astrocyte elevated gene- 1 inhibits growth, induces apoptosis, and increases the chemosensitivity to 5 -fluorouracil in renal cancer Caki-1 cells. Mol Cells 2014; 37: 857-864. http://dx.doi.org/10.14348/ molcells.2014.0081

[36] XU XL, ZENG Y, ZHAO C, HE MZ, WANG F, et al. Salusin- $\beta$ induces smooth muscle cell proliferation by regulating cyclins D1 and E expression through MAPKs signaling pathways. J Cardiovasc Pharmacol 2015; 65: 377-385. http://dx.doi. org/10.1097/FJC.0000000000000209

[37] OHTSUBO M, THEODORAS AM, SCHUMACHER J, ROBERTS JM, PAGANO M. Human cyclin E, a nuclear protein essential for the G1-to-S phase transition. Mol Cell Biol 1995; 15: 2612-2624. http://dx.doi.org/10.1128/ MCB.15.5.2612

[38] RESNITZKY D, REED SI. Different roles for cyclins D1 and $\mathrm{E}$ in regulation of the G1-to-S transition. Mol Cell Biol 1995 Jul; 15: 3463-3469. http://dx.doi.org/10.1128/ MCB.15.7.3463

[39] TANG ZP, CUI QZ, DONG QZ, XU K, WANG EH. Ataxia-telangiectasia group D complementing gene (ATDC) upregulates matrix metalloproteinase 9 (MMP-9) to promote lung cancer cell invasion by activating ERK and JNK pathways. Tumor Biol 2013; 34: 2835-2842. http://dx.doi.org/10.1007/ s13277-013-0843-7

[40] SATO A, NAGASE H, OBINATA D, FUJIWARA K, et al. Inhibition of MMP-9 using a pyrrole-imidazole polyamide reduces cell invasion in renal cell carcinoma. Int J Oncol 2013; 43: 1441-1446.

[41] HIMELSTEIN BP, CANETE-SOLER R, BERNHARD EJ, DILKS DW, MUSCHEL RJ. Metalloproteinases in tumor progression: the contribution of MMP-9. Invasion Metastasis 1994; 14: 246-258. 